\title{
CII. IMPROVEMENTS IN COLORIMETRY.
}

\author{
BY ROBERT VINER STANFORD. \\ From the Chemical Laboratory, Cardiff City Mental Hospital.
}

(Received September 20th, 1923.)

Some years ago I pointed out [Stanford, 1913] that it is not possible to compare the depth of colour of two solutions unless they are of the same shade of colour, and that therefore the colorimetric comparison must be made when the liquids are of the same concentration, and this condition necessarily involves their being of the same thickness of layer. Following this principle, I described in that paper a "dilution" colorimeter, in which the two liquids are contained in similar glass cells, the stronger being then diluted until equality is reached. Whilst that instrument cannot well be criticised from a theoretical point of view, in actual use it is most inconvenient. It is the object of this note to describe improvements which make the dilution colorimeter as rapid and simple to manipulate as any other. These improvements partly concern the colorimeter itself, and partly the source of light employed.

Source of light for colorimetric purposes. The only satisfactory natural source of light for colorimetric purposes is the diffused daylight of a northern sky, but even if this be available its varying intensity is a drawback, because, for any coloured solution, there is a particular intensity of coloration at which a colorimetric comparison is most exact. An unvarying artificial source is therefore preferable.

There is now obtainable under the name of the Sheringham Daylight Lamp a source of light which is identical in quality with the diffused daylight of a northern sky, and does not vary in intensity. Such operations as comparisons in Nessler cylinders can be done with greater ease by this light than by genuine daylight for this reason, and it forms an admirable illuminator for any colour matching instrument.

In this lamp the light from a metallic filament lamp is reflected on to an inverted bowl which is pigmented over strictly proportioned areas with three different pigments, of which a blue predominates. The resulting illumination on a white paper a few inches away is sensibly uniform, and as there is no direct illumination the trouble of actual images does not arise.

Improved dilution colorimeter. The dilution colorimeter in its original form [Stanford, 1913] has the usual pair of rhomboidal prisms, each of which illuminates one-half of the field of view in the eye-piece. In front of the prisms there is a box containing two parallel sided glass cells, one of which 
contains the standard solution, and the other the unknown solution. Over each cell is a burette, so that solvent can be run into the stronger solution until equality of colour is reached. The practical disadvantage of the instrument is the eye strain and waste of time involved in constantly withdrawing the eye from the eye-piece and the hand from the stirrer for the purpose of adding cautiously a drop or two more of solvent. The attachment to be described removes this inconvenience. It is merely necessary to pull a string, and drops of solvent issue from the burette as long as the string is pulled and at a rate previously determined by the observer.

Description. The colorimeter as shown in the accompanying drawings (Figs. 1 and 2, which are to scale) consists of two parts, namely, the light box, $A B D$, and the colorimeter proper.

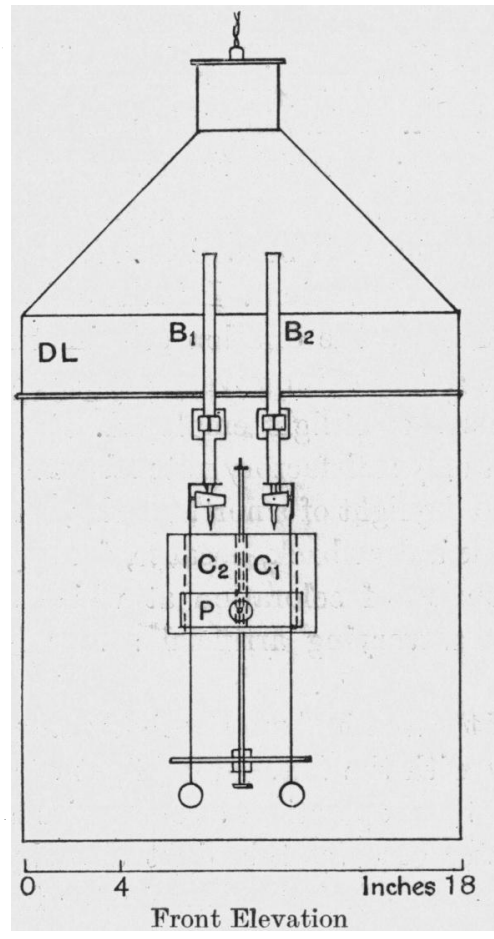

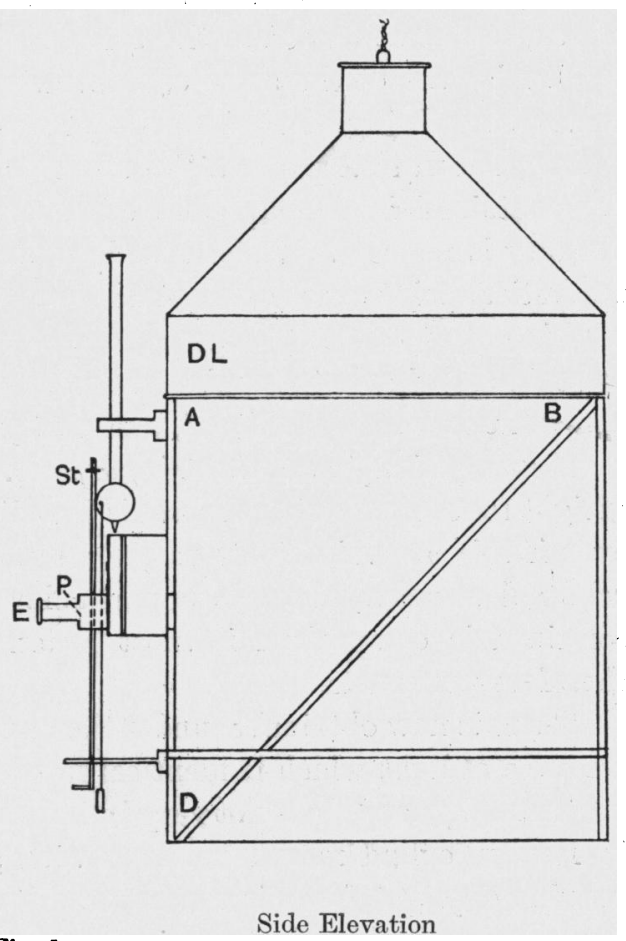

Fig. 1.

The light box consists of a cubical wooden box, each side of which measures $18^{\prime \prime}$, bisected diagonally by another piece of three-ply, shown as $B D$ in the side-elevation. On the top of the box is a Sheringham Daylight Lamp, $D L$. The particular lamp required is known as type $830 \mathrm{~A}$, and has a conical reflector $18^{\prime \prime}$ in diameter.

The semi-cubical space, $A B D$, is lined throughout internally with white drawing paper, and in the front wall, $A D$, has a horizontal slot centrally situated, and measuring $5^{\prime \prime}$ by $1 \frac{1^{\prime \prime}}{}$. 
The colorimeter proper consists of a brass box to hold the two cells $C_{1}$ and $\mathrm{C}_{2}$. This box is screwed to the front of the light box opposite the slot. To the front of this cell-chamber is affixed the case $P$ containing a pair of rhomboidal prisms and the eye-piece $E$. The stirrup-shaped stirrer St (shown with the stirrup removed) passes through the centre of the prism case. It is spring-loaded, and is worked up and down by means of the finger.

Above each cell is a burette, held in a clip so that it can be easily swung to one side to facilitate removal of the glass cell. The special feature of these burettes is the automatic attachment to the tap which enables them to deliver drops of solvent into the cells at any pre-determined rate. This attachment is shown in side and front elevation in the drawing (Fig. 2). A metal plate $C$ is fixed to the stem of the burette below the tap. This acts as a stop. The burette-tap is firmly gripped by two pieces of wood which are centrally attached to a metal disc (the lid of a small tin is convenient) about $2 \frac{1}{2}$ in diameter. Near its edge this disc carries the studs $a, d$ and $e$, which are panelpins held in position with spots of solder, and also the set-pin $b$. The stud $d$ is attached by means of a spring or rubber band to any convenient anchorage. The stud $a$ carries a thread terminating in a ring large enough to admit the finger. The device acts as follows. Normally, the tension of the spring attached to $d$ brings the stud $e$ against the burette, and in this position the tap is closed.

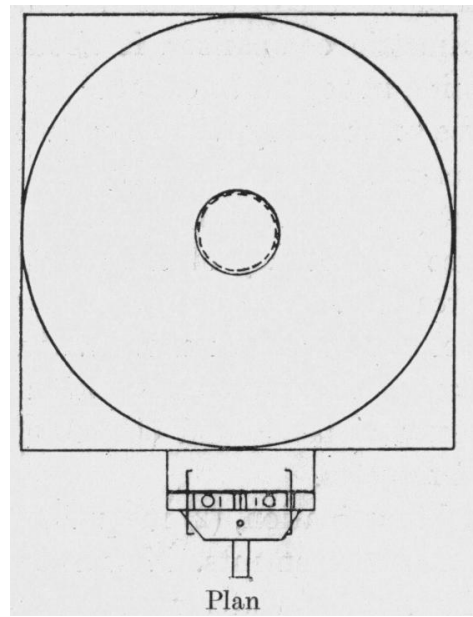

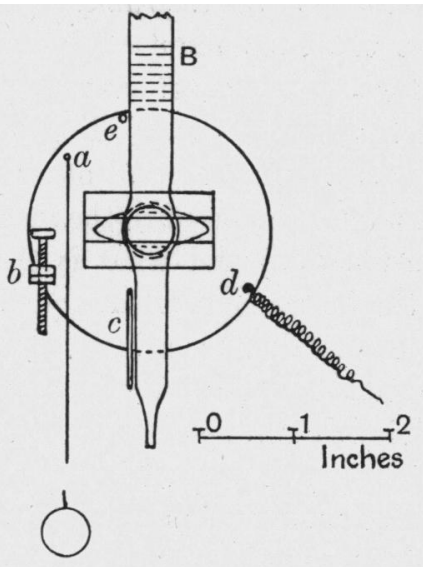

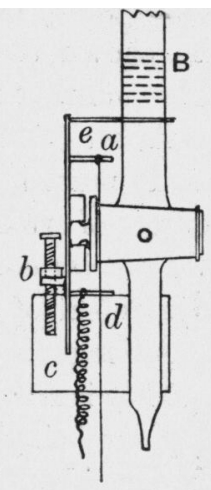

Fig. 2. Improved Dilution Colorimeter.
Automatic Burette Attachment

If now the thread attached to $a$ be pulled down, the tap will open until the end of the set-pin $b$ impinges upon the stop-plate $c$. By suitably adjusting the set-pin, the rate of drops from the end of the burette can be arranged beforehand. A rate of about one in some three seconds will be found convenient.

In using the instrument all that is necessary, therefore, is to place the two cells containing the liquids in position, switch on the daylight lamp and, while continuously working the stirrer with a finger of one hand, to pull the 
string of the burette over the stronger solution, and allow the dilution to proceed until equality of colour is reached. The difference in the readings of the burette gives the volume of solvent added to the stronger solution, and the necessary simple calculation may be made with the help of the formula given below.

Calculation. Let

Total volume of unknown solution be $V_{u}$.

Amount of substance contained therein be $x$.

Volume of solution placed in colorimeter cell be $v_{u}$.

Total volume of standard solution be $V_{s}$.

Amount of substance contained therein be $y$.

Volume of solution placed in colorimeter cell be $v_{s}$.

Volume of water added in dilution be $W$.

Then

A. When standard is diluted:

B. When unknown is diluted:

$$
x=\frac{\mathrm{V}_{u} \times \psi_{\mathrm{a}} \times y}{\boldsymbol{V}_{8} \times\left(v_{s}+W\right)}
$$

$$
x=\frac{V_{u} \times\left(v_{u}+W\right) \times y}{v_{u} \times V_{s}}
$$

Degree of accuracy of the instrument. Some months' use of the instrument has shown that the time required for a colorimetric comparison is about three minutes for any person of average perception in regard to colour.

Apart from this, under the heading of degree of accuracy, the same two points must be dealt with as in all other colorimetric devices, namely, what sources of error may be inherent in the instrument (whether from its principles or from its construction) and what is the degree of accuracy of the actual adjustment of the instrument when equality is reached.

As regards errors due to principles, this colorimeter has none. When equality is reached the liquids under comparison are of the same concentration, they are being viewed through the same thicknesses of layer, and therefore, like Euclid's triangles, they must be equal in all respects.

Under errors of construction come (1) unequal illumination, (2) inequality of the cells, and (3) lack of symmetry of the optical arrangements. Now as to these, the daylight lamp arrangement described entirely obviates unevenness of illumination, which is a fruitful source of trouble in colorimetry as a rule. The internal thicknesses of the cells are tested beforehand by dusting them with lycopodium powder and focussing a measuring microscope first on one surface and then on the other. The two cells should not differ by more than $0.1 \%$. The symmetry of the optical arrangements is controlled by examining in the colorimeter a number of liquids of different colours, a portion of each being put into each cell. In this connection it may be remarked that the only trouble likely to be met with is an unsymmetrical mounting, or a subsequent displacement, of the rhomboidal prisms. 
As a result of a considerable number of experiments, it may be stated that a person with an average eye may expect to get results correct to $1 \%$, provided the comparisons are undertaken at the most sensitive depth of colour for the particular liquids under examination. [Compare Horn, 1906; Horn and Blake, 1906.] This refers to rapid and solitary estimations. If circumstances permit, say, three independent comparisons to be made, the error of the mean will be considerably less than this.

Mechanical stirrer. The simple form of hand stirrer described in connection with the colorimeter answers very well when only a few estimations have to be made, but becomes tedious and fatiguing if the instrument is in constant use. For this reason a mechanical stirrer is an advantage. It is clear that such a stirrer must be instantly detachable, for both it and the cell in which it works have to be cleaned and dried after each estimation.

Our stirrer is made from a dentist's drilling engine, which consists of a flexible shaft, passing through a flexible cable and carrying at one end a pulley and at the other end a spring bayonet arrangement by means of which the shaft can be instantly connected with the so-called "hand piece." This hand piece contains a small chuck for holding the drills. The actual stirrer is a piece of $\frac{3}{16}$ " glass rod flattened and twisted at the lower end to form a kind of screw, and mounted at its upper end in a socket soldered to an ordinary dental drill. The hand piece is rigidly fixed on a T-shaped piece of wood which slides in two wooden grooves screwed to the front of the light box. When the cells are in place, the piece of wood carrying the handpiece and stirrer is dropped into its slots, the end of the cable pushed on to the bayonet joint, and everything is ready for the estimation. The stirrer is driven by any convenient small motor.

\section{REFERENCES.}

Horn (1906). Amer. Chem. J. 35, 253.

Horn and Blake (1908). Amer. Chem. J. 36, 195 and 516.

Stanford (1913). Z. physiol. Chem. 87, 159; Reports from the Chemical Laboratory, Cardiff City Mental Hospital, No. 3. 